\title{
YIELD QUALITY OF SOLANUM TUBEROSUM L. UNDER THE INFLUENCE OF MICROBIAL PREPARATIONS AND DEPENDING ON PLANTING TERMS IN WESTERN POLISSYA REGION
}

Boroday V. V. ${ }^{1}$, Danilkova T. V. ${ }^{2}$, Koltunov V. A. ${ }^{3}$

${ }^{1}$ National University of Life and Environmental Sciences of Ukraine, 13, Heroiv Oborony Str., Kyiv, 03041, E-mail: veraboro@gmail.com

${ }^{2}$ State Phytosanitary Inspection of Lviv Region, 18, Vitovskogo Str., 79011, Lviv

${ }^{3}$ Kyiv National University of Trade and Economics, 19 Kyoto Str., 02156, Kyiv

Application of microbial preparation Planrhiz and fungicide Rydomil Gold MC 68 WG have improved yield, marketability of potatoes and have increased the standard part of tubers. Plants bakterization have promoted increase of potatoes marketability by reducing the number of small, mechanically damaged and sick tubers, with the crop yield higher than the control in 1,5-1,9 times while the number of sick tubers have decreased in 2,4-5,5 times. The combination of microbial agents (Planrhiz + Diazofit + Phosphoenteryn) compared to single application of Planrhiz have revealed no significant difference between the yield and product marketability. As for the planting terms - the 1st planting term in the third decade of April have showed to be the best in West Polissya due to the formation of bigger number of standard part of the tubers.

Keywords: Solanum tuberosum L., microbial preparations, marketability, grade-out crop, quality, planting terms.

Commercial quality of the products, designed for long - term storage, sale and processing, is largely determined by crop cultivation and harvesting conditions $[3,6,10]$. Potatoes are very sensitive biological object actively responding to the external influences that directly affect its consumer characteristics. Stability during storage of vegetables and potatoes, their nutritional value, marketability and value are specified by a set of interrelated factors throughout their way to the consumer. However, in Ukraine, this integrated technological circuit is divided into the three weakly interconnected stages: production, storage and sales. So, quality preservation and loss reduction of fresh vegetables and potatoes during growing, harvesting, transportation, storage and sales is a complex problem that is of great importance. Unfavorable abiotic factors affect the quality of crop production, causing decrease in resistance of vegetables and potatoes to fungal, bacterial and viral diseases. Epiphytotic nature of their spread and products damage during growing, storage and sales requires improvement of cultivation and storage technologies of potatoes.

With the constant rise of costs, a radical change in the technologies of potato growing is possible only through the development of adaptive farming systems, based on the use of energy - saving, environmentally friendly, biological technologies. Moreover, the most effective element in the structure of the processing methods aimed at improving the productivity of potato is the use of high - yielding varieties, which biological features meet the specific growing 
conditions $[3,9,10]$. In this regard, it should be noted that current assortment of potatoes varieties do not fully satisfy the requirements and needs of producers. Besides, the agroecological assessment of varieties is absent or is not performed at all $[3,11]$. Therefore, along with the need to create new high - yielding varieties, it is expedient to develop complex farming practices that would promote implementation of potential characteristics of varieties in different agro - climatic conditions.

The objective of given study was to identify the cultivation conditions and processing methods under which the minimum number of grade - out and marketable potato tubers will be produced.

Materials and methods. Methods of field experiments and research in storing potatoes in given studies are commonly used [2, 8, 10]. Biological preparations Planrhiz (based on bacteria Pseudomonas fluorescens AR - 33), Diazofit (biological agent - Agrobacterium radiobacter), Phosphoenteryn (PMB) (based on phosphorous mobilizing bacteria Enterobacter nimipressuralis 32-3), produced in the biological laboratory of State Inspection for Plant Protection in Lviv region were used. Potatoes (early varieties Skarbnitsya and middle - ripening Lileya), due to the unfavorable rainy conditions in Lviv area, were planted in spring in the third week of April, and in the second and third weeks of May. Crop harvesting was performed during third week of August and second week of September.

Results and discussion. Application of biological and chemical preparations compared with control variants have increased productivity, improving marketability of potatoes and reducing the number of grade - out tubers.

The highest yield was formed by Skarbnitsya variety at complex application of Planrhiz + Diazofit + FMB $(2.0+0.2+0.2$ 1/ha $)-36,2-48,3 \mathrm{t} / \mathrm{ha}$. For Lileya varieties - at use of Rydomil Gold (27,36 - 47,48 t/ha) (Tables 1, 2, 3, 4). However, the highest marketability of Lileya variety was in variants with Planrhiz application $(2.51 / \mathrm{ha}$ ) $-83,8-90 \%$ (variant without treatment $-57,5-71,3 \%$, at application of Rydomil Gold - 73.9-86.9 \%). Potatoes yield at use of Planrhiz in different doses in Lileya variety - was $24,64-41,78 \mathrm{t} /$ ha for joint inoculation with Planrhiz + Diazofit + FMB - 24,08 - 43,68 t/ha; in Skarbnitsya variety - 30, $24-$ $28,2-48,30$ and $40.7 \mathrm{t} / \mathrm{ha}$, respectively.

The use of microbial preparations have contributed to an output of more marketable tubers. Grade-out crop was smaller comparing to the control due to the formation of a small number of tubers damaged by disease, mechanically damage and small tubers. Among all grade-out harvest components the part of small tubers was $45.1 \%$, affected by diseases $-30.6 \%$ and mechanically damaged $-16.0 \%$. Thus, for Lileya variety of first planting term the percentage of grade-out crops was $10 \%$ at application of Planrhiz $(2.5 \mathrm{l} / \mathrm{ha}$ ) (control without treatment $-28.7 \%$ ), at this the lowest yield of sick and affected by diseases tubers (3 9 and $2.5 \%$ vs. 8.9 and $11.7 \%$ in control) was observed (Table 1). A similar dependences were observed for the combined application of Planrhiz + Diazofit + FMB $(2.5+0.2$ $+0.21 /$ ha $)-8.5$ and $3.3 \%$ of small and affected by diseases tubers vs. 16.5 and $14.7 \%$ in control, respectively. As for Skarbnitsya variety of first planting term the 
marketable tuber yield was $25.3 \%$ in the control, $10.8 \%$ - at Planrhiz application $(2.5 \mathrm{l} / \mathrm{ha})$ and $10.1 \%$ - at combined use of Planrhiz + Diazofitom + FMB (1.5 + $0.2+0.21 /$ ha) (Table 3).

During three years of investigation in all four soil-climatic zones of Lviv region - the highest total and marketable tuber yield was obtained from the first planting period, i.e. in the third decade of April $[1,5,7]$. The third term - the third decade of May - was hardly applicable. Shifting the planting terms into the period with intense intake of PAR and heat improves some conditions of photosynthetic activity of potato crops, but leads to the diseases development, reduction of leaf surface, chlorophyll formation, as well as accumulation of yield and starch in tubers. Thus, the yield obtained in variants with Lileya varieties of first planting term have exceeded the yield of the second planting term in average on 18,3-42,4 $\%$; for Skarbnitsya varieties - on 11,2-36,7\%; yield of marketable products was higher in Lileya varieties on $27.7-50.9 \%$ and Skarbnitsya varieties - on 15,2$41,9 \%$. The duration of the growing season was determined by the biological characteristics of the potato varieties. By the changes in the studied parameters the early variety Skarbnitsya was relatively stable as compared to the middle ripening Lileya variety. In the first planting term the quality grades evaluation for Lileya and Skarbnitsya varieties have not revealed any significant differences, while at the second -Skarbnitsya variety plants had yield higher on $4.57 \mathrm{t} / \mathrm{ha}$, structure almost no differences at grade-out products structure.

Biopreparations have contributed in a certain extent to the ecological plasticity and adaptation of Lileya variety. In control variant (without treatment) of first planting terms the marketable yield was $17.14 \mathrm{t} / \mathrm{ha}$, and $8.65 \mathrm{t} / \mathrm{ha}$ - in the second (less than 2 times), while application of microbial preparations have leveled that difference - on average the output marketability have decreased on 27,7$39,5 \%$.

At combined use of Planrhiz and Gold Rydomil at first planting terms, no difference between the output quality was observed. However, the second planting term if compared to only single fungicide treatment in a Lileya variety the yield was higher on $5.22 \mathrm{t} / \mathrm{ha}$, with $7.7 \%$ increase of marketability, and $4.8 \%$ decrease of small tubers yield and $3.3 \%$ - of tubers affected by diseases (Table 2). For Skarbnitsya variety, these values had no significant differences (Table 4).

Among the studied concentrations of Planrhiz, application of $2.0-2.51$ / ha as comparing to $1.0-1.51 /$ ha have showed the best results, while at combined use of Planrhiz with Diazofit and FMB $-2.5+0.2+0.21 /$ ha, respectively.

Some researchers state that combination of preparations subject to the environmental and physiological compatibility of bacteria may differ in stability and efficiency in different agro-climatic conditions [4, 9]. However, in Western Polissya, the combination of microbial preparations as compared to single application of Planrhiz have revealed no significant difference between the yield and marketability indices, as wells as between the number of small tubers and tubers affected by diseases. 
TABLE 1. Structure of potato crop yield, depending on planting terms and application of biological and chemical preparations (2009-2011, Lileya variety, West Polissya, first planting term)

\begin{tabular}{|c|c|c|c|c|c|c|c|c|c|c|}
\hline \multirow[b]{3}{*}{ Variants } & \multicolumn{3}{|c|}{ Yield } & \multicolumn{7}{|c|}{ Grade-out yield part } \\
\hline & \multirow[b]{2}{*}{$\begin{array}{c}\text { Total, } \\
\text { t/ha }\end{array}$} & \multirow[b]{2}{*}{$\begin{array}{l}\text { Commer- } \\
\text { cial, } t / \text { ha }\end{array}$} & \multirow[b]{2}{*}{$\begin{array}{l}\text { Marke- } \\
\text { tability, } \\
\quad \%\end{array}$} & \multicolumn{2}{|c|}{ Total } & \multicolumn{5}{|c|}{ including, $\%$} \\
\hline & & & & $\mathrm{t} / \mathrm{ha}$ & $\%$ & small & $\begin{array}{l}\text { with } \\
\text { emergences, } \\
\text { greenish }\end{array}$ & $\begin{array}{l}\text { mecha- } \\
\text { nically } \\
\text { damaged }\end{array}$ & $\begin{array}{l}\text { affected } \\
\text { by pests }\end{array}$ & $\begin{array}{l}\text { affected } \\
\text { by } \\
\text { diseases }\end{array}$ \\
\hline W/o treatment (control) & 24.04 & 17.14 & 71.3 & 6.90 & 28.7 & 8.9 & 0.5 & 4.0 & 3.1 & 11.7 \\
\hline Phytocide. 11/ ha & 37.00 & 32.04 & 86.6 & 4.96 & 13.4 & 4.6 & 0.0 & 1.8 & 0.0 & 6.4 \\
\hline $\begin{array}{l}\text { Rydomil Gold MC } 68 \text { WG } \\
\text { c.g }\end{array}$ & 47.48 & 41.25 & 86.9 & 6.23 & 13.1 & 5.6 & 0.0 & 2.1 & 2.3 & 4.7 \\
\hline Planrhiz (1.0 1/ ha) & 37.48 & 31.04 & 82.8 & 6.44 & 17.2 & 9.6 & 1.0 & 1.9 & 0.8 & 4.0 \\
\hline Planrhiz (1.5 l / ha) & 36.70 & 31.74 & 86.5 & 4.96 & 13.5 & 4.4 & 0.0 & 2.4 & 1.5 & 4.5 \\
\hline Planrhiz (2.0 1/ ha) & 38.26 & 32.86 & 85.9 & 5.40 & 14.1 & 7.7 & 0.5 & 0.9 & 0.6 & 4.1 \\
\hline Planrhiz (2.5 1 / ha) & 41.78 & 37.60 & 90.0 & 4.18 & 10.0 & 3.9 & 0.5 & 2.5 & 1.0 & 2.5 \\
\hline $\begin{array}{l}\text { Planrhiz + Diazofit + PMB } \\
(1.0+0.2+0.21 / \text { ha })\end{array}$ & 32.76 & 28.96 & 88.4 & 3.81 & 11.6 & 5.4 & 0.5 & 2.6 & 1.7 & 1.7 \\
\hline $\begin{array}{l}\text { Planrhiz + Diazofit + PMB } \\
(1.5+0.2+0.21 / \mathrm{ha})\end{array}$ & 36.32 & 31.14 & 85.7 & 5.18 & 14.3 & 10.7 & 0.0 & 2.9 & 0.3 & 2.3 \\
\hline $\begin{array}{l}\text { Planrhiz + Diazofit + PMB } \\
(2.0+0.2+0.21 / \text { ha })\end{array}$ & 41.53 & 36.97 & 89.0 & 4.57 & 11.0 & 5.5 & 0.0 & 2.7 & 0.2 & 2.7 \\
\hline $\begin{array}{l}\text { Planrhiz + Diazofit + PMB } \\
(2.5+0.2+0.21 / \mathrm{ha})\end{array}$ & 43.68 & 39.12 & 89.6 & 4.56 & 10.4 & 5.7 & 0.4 & 1.7 & 0.0 & 2.1 \\
\hline $\begin{array}{l}\text { Planrhiz+ Rydomil Gold } \\
\text { MC } 68 \text { WG, c. g( } 2.0+2.51 / \\
\text { ha) }\end{array}$ & 43.86 & 37.32 & 85.1 & 6.54 & 14.9 & 5.9 & 0.0 & 2.7 & 0.7 & 5.5 \\
\hline $\mathrm{LSD}_{05}$ & $\begin{array}{c}1.2- \\
1.4\end{array}$ & & & & & & & & & \\
\hline Mean & 38.41 & 33.10 & 85.6 & 5.31 & 14.4 & 6.5 & 0.3 & 2.3 & 1.0 & 4.4 \\
\hline
\end{tabular}

Note: no faded, frostbitten or stewed tubers, as well as soil were present. 
TABLE 2. Structure of potato crop yield, depending on planting terms and application of biological and chemical preparations (2009-2011 biennium, Lileya variety, West Polissya, second planting term)

\begin{tabular}{|c|c|c|c|c|c|c|c|c|c|c|}
\hline \multirow[b]{3}{*}{ Variants } & \multicolumn{3}{|c|}{ Yield } & \multicolumn{7}{|c|}{ Grade-out yield part } \\
\hline & \multirow[b]{2}{*}{$\begin{array}{c}\text { Total, } \\
\text { t/ha }\end{array}$} & \multirow[b]{2}{*}{$\begin{array}{l}\text { Commer- } \\
\text { cial, } t / \text { ha }\end{array}$} & \multirow[b]{2}{*}{$\begin{array}{l}\text { Marke- } \\
\text { tability, } \\
\quad \%\end{array}$} & \multicolumn{2}{|c|}{ Total } & \multicolumn{5}{|c|}{ including, $\%$} \\
\hline & & & & $\mathrm{t} / \mathrm{ha}$ & $\%$ & small & $\begin{array}{l}\text { with } \\
\text { emergences, } \\
\text { greenish }\end{array}$ & $\begin{array}{c}\text { mecha- } \\
\text { nically } \\
\text { damaged }\end{array}$ & $\begin{array}{l}\text { affected } \\
\text { by pests }\end{array}$ & $\begin{array}{l}\text { affected } \\
\text { by } \\
\text { diseases }\end{array}$ \\
\hline W/o treatment (control) & 15,06 & 8,65 & 57,5 & 6,41 & 42,5 & 16,5 & 0,0 & 6,5 & 4,4 & 14,7 \\
\hline Phytocide. 11 / ha & 25,94 & 19,69 & 75,9 & 6,25 & 24,1 & 9,0 & 0,5 & 5,0 & 2,3 & 8,3 \\
\hline $\begin{array}{l}\text { Rydomil Gold MC } 68 \text { WG } \\
\text { c.g }\end{array}$ & 27,36 & 20,22 & 73,9 & 7,14 & 26,1 & 15,3 & 0,3 & 2,4 & 1,5 & 8,4 \\
\hline Planrhiz (1.0 l / ha) & 24,64 & 19,53 & 79,3 & 5,11 & 20,7 & 10,3 & 0,0 & 2,8 & 1,3 & 7,8 \\
\hline Planrhiz (1.5 l / ha) & 30,00 & 22,93 & 76,4 & 7,07 & 23,6 & 14,7 & 0,5 & 2,6 & 2,2 & 5,3 \\
\hline Planrhiz (2.0 l / ha) & 26,06 & 20,56 & 78,9 & 5,50 & 21,1 & 14,2 & 0,7 & 2,8 & 1,7 & 4,1 \\
\hline Planrhiz (2.5 1/ ha) & 30,00 & 25,13 & 83,8 & 4,87 & 16,2 & 8,4 & 0,6 & 2,4 & 0,2 & 4,8 \\
\hline $\begin{array}{l}\text { Planrhiz + Diazofit + PMB } \\
(1.0+0.2+0.21 / \text { ha })\end{array}$ & 25,96 & 20,93 & 80,6 & 5,03 & 19,4 & 14,2 & 0,0 & 2,5 & 0,3 & 5,9 \\
\hline $\begin{array}{l}\text { Planrhiz + Diazofit + PMB } \\
(1.5+0.2+0.21 / \text { ha })\end{array}$ & 24,08 & 18,85 & 78,3 & 5,23 & 21,7 & 24,7 & 0,0 & 0,6 & 0,0 & 4,5 \\
\hline $\begin{array}{l}\text { Planrhiz + Diazofit + PMB } \\
(2.0+0.2+0.21 / \text { ha })\end{array}$ & 30,16 & 25,33 & 84,0 & 4,83 & 16,0 & 7,9 & 0,0 & 3,1 & 1,7 & 4,4 \\
\hline $\begin{array}{l}\text { Planrhiz + Diazofit + PMB } \\
(2.5+0.2+0.21 / \text { ha })\end{array}$ & 31,14 & 27,03 & 86,8 & 4,11 & 13,2 & 8,5 & 0,0 & 1,4 & 0,3 & 3,3 \\
\hline $\begin{array}{l}\text { Planrhiz+ Rydomil Gold } \\
\text { MC } 68 \text { WG, c. g( } 2.0+2.51 / \\
\text { ha) }\end{array}$ & 32,58 & 26,53 & 81,6 & 6,00 & 18,4 & 10,5 & 0,0 & 3,6 & 1,6 & 5,1 \\
\hline $\mathrm{LSD}_{05}$ & $\begin{array}{c}1,3- \\
1,5 \\
\end{array}$ & & & & & & & & & \\
\hline Mean & 26,92 & 21,20 & 78,1 & 5,63 & 21,9 & 12,8 & 0,2 & 3,0 & 1,5 & 6,4 \\
\hline
\end{tabular}


TABLE 3. Structure of potato crop yield, depending on planting terms and application of biological and chemical preparations (2009-2011 biennium, Skarbnitsya variety, West Polissya, first planting term)

\begin{tabular}{|c|c|c|c|c|c|c|c|c|c|c|}
\hline \multirow[b]{3}{*}{ Variants } & \multicolumn{3}{|c|}{ Yield } & \multicolumn{7}{|c|}{ Grade-out yield part } \\
\hline & \multirow[b]{2}{*}{$\begin{array}{c}\text { Total, } \\
\text { t/ha }\end{array}$} & \multirow[b]{2}{*}{$\begin{array}{l}\text { Commer- } \\
\text { cial, } t / \text { ha }\end{array}$} & \multirow[b]{2}{*}{$\begin{array}{l}\text { Marke- } \\
\text { tability, } \\
\quad \%\end{array}$} & \multicolumn{2}{|c|}{ Total } & \multicolumn{5}{|c|}{ including, \% } \\
\hline & & & & $\mathrm{t} / \mathrm{ha}$ & $\%$ & small & $\begin{array}{c}\text { with } \\
\text { emergences, } \\
\text { greenish }\end{array}$ & $\begin{array}{c}\text { mecha- } \\
\text { nically } \\
\text { damaged }\end{array}$ & $\begin{array}{l}\text { affected } \\
\text { by pests }\end{array}$ & $\begin{array}{l}\text { affected } \\
\text { by } \\
\text { diseases }\end{array}$ \\
\hline W/o treatment (control) & 25,72 & 19,20 & 74,7 & 6,52 & 25,3 & 8,3 & 0,3 & 4,3 & 1,9 & 10,4 \\
\hline Phytocide. 11 / ha & 42,30 & 36,29 & 85,8 & 6,01 & 14,2 & 5,1 & 0,7 & 1,5 & 2,2 & 4,6 \\
\hline $\begin{array}{l}\text { Rydomil Gold MC } 68 \text { WG } \\
\text { c.g }\end{array}$ & 41,14 & 36,15 & 87,9 & 4,99 & 12,1 & 5,5 & 0,0 & 3,2 & 1,1 & 2,3 \\
\hline Planrhiz (1.0 1/ ha) & 35,82 & 30,39 & 84,8 & 5,43 & 15,2 & 6,6 & 0,3 & 2,6 & 1,9 & 3,8 \\
\hline Planrhiz (1.5 1/ ha) & 40,70 & 34,86 & 85,7 & 5,84 & 14,3 & 5,3 & 0,0 & 3,6 & 1,9 & 3,4 \\
\hline Planrhiz (2.0 1 / ha) & 37,50 & 32,20 & 85,9 & 5,30 & 14,1 & 7,3 & 0,0 & 1,5 & 2,5 & 2,9 \\
\hline Planrhiz (2.5 1/ ha) & 39,28 & 35,05 & 89,2 & 4,23 & 10,8 & 4,0 & 0,0 & 1,3 & 2,7 & 2,8 \\
\hline $\begin{array}{l}\text { Planrhiz + Diazofit + PMB } \\
(1.0+0.2+0.21 / \text { ha })\end{array}$ & 44,52 & 38,94 & 87,5 & 5,58 & 12,5 & 4,3 & 0,0 & 2,9 & 2,6 & 2,3 \\
\hline $\begin{array}{l}\text { Planrhiz + Diazofit + PMB } \\
(1.5+0.2+0.21 / \text { ha })\end{array}$ & 43,08 & 38,72 & 89,9 & 4,36 & 10,1 & 4,4 & 0,3 & 2,0 & 0,7 & 2,5 \\
\hline $\begin{array}{l}\text { Planrhiz + Diazofit + PMB } \\
(2.0+0.2+0.21 / \text { ha })\end{array}$ & 48,30 & 41,48 & 85,9 & 6,82 & 14,1 & 7,4 & 0,0 & 1,6 & 0,5 & 4,4 \\
\hline $\begin{array}{l}\text { Planrhiz + Diazofit + PMB } \\
(2.5+0.2+0.21 / \text { ha })\end{array}$ & 46,14 & 40,07 & 86,8 & 6,07 & 13,2 & 9,1 & 0,0 & 1,8 & 0,9 & 1,9 \\
\hline $\begin{array}{l}\text { Planrhiz+ Rydomil Gold } \\
\text { MC 68WG, c. g( } 2.0+2.51 / \\
\text { ha) }\end{array}$ & 40,14 & 35,40 & 88,2 & 4,74 & 11,8 & 4,7 & 0,0 & 2,7 & 1,2 & 3,5 \\
\hline $\mathrm{LSD}_{05}$ & $\begin{array}{c}1,1- \\
1,4\end{array}$ & & & & & & & & & \\
\hline Mean & 40,39 & 34,90 & 86,0 & 5,49 & 14,0 & 6,0 & 0,1 & 2,4 & 1,7 & 3,7 \\
\hline
\end{tabular}


TABLE 4. Structure of potato crop yield, depending on planting terms and application of biological and chemical preparations (2009-2011 biennium, Skarbnitsya variety, West Polissya, second planting term)

\begin{tabular}{|c|c|c|c|c|c|c|c|c|c|c|}
\hline \multirow[b]{3}{*}{ Variants } & \multicolumn{3}{|c|}{ Yield } & \multicolumn{7}{|c|}{ Grade-out yield part } \\
\hline & \multirow[b]{2}{*}{$\begin{array}{c}\text { Total, } \\
\text { t/ha }\end{array}$} & \multirow[b]{2}{*}{$\begin{array}{l}\text { Commer- } \\
\text { cial, } t / \text { ha }\end{array}$} & \multirow[b]{2}{*}{$\begin{array}{l}\text { Marke- } \\
\text { tability, } \\
\quad \%\end{array}$} & \multicolumn{2}{|c|}{ Total } & \multicolumn{5}{|c|}{ including, $\%$} \\
\hline & & & & $\mathrm{t} / \mathrm{ha}$ & $\%$ & small & $\begin{array}{l}\text { with } \\
\text { emergences, } \\
\text { greenish }\end{array}$ & $\begin{array}{c}\text { mecha- } \\
\text { nically } \\
\text { damaged }\end{array}$ & $\begin{array}{l}\text { affected } \\
\text { by pests }\end{array}$ & $\begin{array}{l}\text { affected } \\
\text { by } \\
\text { diseases }\end{array}$ \\
\hline W/o treatment (control) & 22,84 & 16,29 & 71,3 & 6,55 & 28,7 & 12,2 & 0,3 & 2,9 & 2,0 & 10,9 \\
\hline Phytocide. 11 / ha & 27,44 & 21,07 & 76,8 & 6,37 & 23,2 & 18,0 & 0,0 & 1,7 & 0,7 & 7,8 \\
\hline $\begin{array}{l}\text { Rydomil Gold MC } 68 \text { WG } \\
\text { c.g }\end{array}$ & 28,34 & 23,31 & 82,2 & 5,04 & 17,8 & 10,0 & 0,0 & 2,6 & 1,2 & 4,3 \\
\hline Planrhiz (1.0 l / ha) & 30,24 & 23,55 & 77,9 & 6,69 & 22,1 & 12,3 & 0,0 & 1,9 & 2,1 & 6,5 \\
\hline Planrhiz (1.5 l / ha) & 34,54 & 27,94 & 80,9 & 6,60 & 19,1 & 8,2 & 0,3 & 4,2 & 1,7 & 5,3 \\
\hline Planrhiz (2.0 l / ha) & 36,64 & 28,67 & 78,3 & 7,97 & 21,7 & 11,1 & 0,7 & 2,5 & 0,9 & 7,1 \\
\hline Planrhiz (2.5 1/ ha) & 31,74 & 24,96 & 78,6 & 6,78 & 21,4 & 11,0 & 0,0 & 4,1 & 2,3 & 5,1 \\
\hline $\begin{array}{l}\text { Planrhiz + Diazofit + PMB } \\
(1.0+0.2+0.21 / \text { ha })\end{array}$ & 28,20 & 22,84 & 81,0 & 5,36 & 19,0 & 9,4 & 0,5 & 1,7 & 1,4 & 7,1 \\
\hline $\begin{array}{l}\text { Planrhiz + Diazofit + PMB } \\
(1.5+0.2+0.21 / \text { ha })\end{array}$ & 32,14 & 25,95 & 80,7 & 6,19 & 19,3 & 7,5 & 0,3 & 4,6 & 2,2 & 5,5 \\
\hline $\begin{array}{l}\text { Planrhiz + Diazofit + PMB } \\
(2.0+0.2+0.21 / \text { ha })\end{array}$ & 36,20 & 29,24 & 80,8 & 6,96 & 19,2 & 9,1 & 0,0 & 2,0 & 2,5 & 6,3 \\
\hline $\begin{array}{l}\text { Planrhiz + Diazofit + PMB } \\
(2.5+0.2+0.21 / \text { ha })\end{array}$ & 34,04 & 27,12 & 79,7 & 6,92 & 20,3 & 10,3 & 0,0 & 3,0 & 3,0 & 5,2 \\
\hline $\begin{array}{l}\text { Planrhiz+ Rydomil Gold } \\
\text { MC } 68 \text { WG, c. g( } 2.0+2.51 / \\
\text { ha) }\end{array}$ & 34,59 & 27,51 & 79,5 & 7,08 & 20,5 & 10,5 & 0,5 & 5,0 & 1,3 & 4,2 \\
\hline $\mathrm{LSD}_{05}$ & $\begin{array}{c}1,2- \\
1,5 \\
\end{array}$ & & & & & & & & & \\
\hline Mean & 31,49 & 24,87 & 79,0 & 6,54 & 21,0 & 10,8 & 0,2 & 3,0 & 1,8 & 6,3 \\
\hline
\end{tabular}


Thus, in Western Polessye, as compared to the control variants (water treatment) and biological control (Fitocide), the biological preparation Planrhiz and its combined use with Diazofit and FMB in different concentrations had influenced on many factors, including yield increase in 1,5-1 9 times, output of standard tubers parts in 1.2-1.4 times and reduction of small, mechanically damaged and sick tubers in 2,4-5,5 times. When storing it is important to determine not only the number of grade-out tubers, but also its structure. Treatment of potatoes with Planrhiz has resulted in the highest output of standard products due to reduction of small, mechanically damaged and sick tubers. The combined use of biological preparations Planrhiz + Diazofit + FMB had the same effect. Potato treatment with the mixture of Planrhiz and Rydomilom Gold MC $68 \mathrm{WG}$ was proved to be more effective than the single fungicide use during the second planting term. In terms of planting in Western Polissya area the best results were obtained in variants with the first planting term. 